M Pai (Hamilton); J Teitel, B Garvey, V Blanchette (Toronto); A Giles, D Lillicrap (Kingston); J Drouin, B Luke (Ottawa); J Dahmer (Sudbury); J Herst (Thunder Bay); M LepineMartin (Sherbrooke); G Rivard, H Strawczynski (Montreal); F Jobin, C Demers (Quebec); K Ali (St John's); S Robinson (Halifax); S Rubin (Moncton); E Ross (Charlottetown).

We thank the nurse coordinators and clinic secretaries for collecting data; Professor Robin Roberts for statistics; the Canadian Red Cross Blood Transfusion Service (S McGuire) for information concerning blood products; and the Computation Services Unit, McMaster University. Funding was provided by the Canadian Hemophilia Society and the
Federal Centre for AIDS, Health Protection Branch, Health and Welfare Canada.

1 Madhok R, Forbes CD. HIV-1 infection in haemophilia. Baillieres Clin Haematol 1990;3:79-101.

2 Prince AM, Horowitz B, Horowitz MS, Zang E. The development of virus-free labile blood derivatives-a review. Eur f Epidemiol 1987;3:103-18.

3 Walker IR. Survey of the Canadian hemophilia population. Can 7 Public Health 1991:82:127-9.

4 AIDS Group of the United Kingdom Haemophilia Centre Directors. Prevalence of antibody to HTLV-III in haemophiliacs in the United Kingdom. BMF 1986;293:175-6.

(Accepted 20 October 1992)

\title{
Time of presentation, time of operation, and unnecessary appendicectomy
}

\author{
A D McLean, P A Stonebridge, A W Bradbury, \\ J B Rainey, D A D Macleod
}

\section{Department of Surgery, St John's Hospital, Livingston, West Lothian EH54 6PP \\ A D McLean, senior house officer \\ P A Stonebridge, senior registrar \\ A W Bradbury, lecturer \\ J B Rainey, consultant surgeon D A D Macleod, consultant surgeon}

Correspondence to: Mr P A Stonebridge, Department of Surgery, Royal Infirmary, Edinburgh EH3 9YW.
The correct diagnosis of acute appendicitis depends on clinical acumen and experience. ${ }^{12}$ Our aim in this study was to establish whether the rate of unnecessary operations and the rate of complicated appendicitis among patients undergoing surgery for suspected acute appendicitis were related to the time of presentation to hospital and the delay between presentation and surgery.

\section{Patients, methods, and results}

We reviewed the case notes of 578 of the 587 patients who underwent emergency surgery for suspected appendicitis between 1 January 1986 and 31 December 1990. The time of presentation (from computer generated accident and emergency sheets), time of operation (from the anaesthetic record), delay to surgery (calculated from the above), and final diagnosis (based on independent histopathological examination) were recorded. An unnecessary operation was deemed to be one that was performed without pathological evidence of surgically remediable disease. Complicated appendicitis was defined as appendicitis with histopathological evidence of perforation or gangrenous change.

Altogether 347 patients had uncomplicated appendicitis, 73 had complicated appendicitis, 14 had other surgical disease requiring operation, and $144 \mathrm{had}$ an unnecessary operation. There was no significant relation between the time from presentation to operation and the proportions of patients with complicated appendicitis and of unnecessary operations, although $39 \%$ of operations performed within two hours of presentation were unnecessary. Patients who presented between midnight and 6 am had a higher rate of unnecessary operations $(30 / 83,36 \%)$ than those who presented at other times $\left(x^{2}, \mathrm{p}<0.05\right)$ : $21 / 118(18 \%)$ presenting between 6 am and noon, $50 / 192(26 \%)$ presenting between noon and $6 \mathrm{pm}$, and $43 / 185(23 \%)$ presenting between $6 \mathrm{pm}$ and midnight. The rate of unnecessary operations in patients who presented between midnight and 6 am was highest in those who underwent surgery within three hours $(18 / 39,46 \%)$ (table). Patients who were operated on between midnight and $6 \mathrm{am}$ also had an increased rate of unnecessary operations (39/125, $31 \%)$.

There was no relation between the rate of complicated appendicitis and time of presentation (six (7\%)
Number (percentage) of unnecessary operations in relation to time of presentation and time to operation

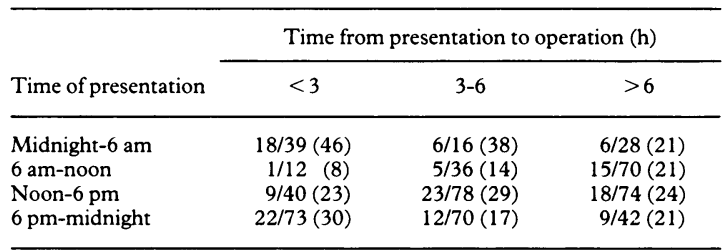

patients presenting between midnight and 6 am had complicated appendicitis, $13(11 \%)$ presenting between 6 am and noon, $33(17 \%)$ presenting between noon and $6 \mathrm{pm}$, and $21(11 \%)$ presenting between $6 \mathrm{pm}$ and midnight) or time of operation.

\section{Comment}

Our study reinforces the report of the National Confidential Enquiry into Perioperative Deaths, ${ }^{3}$ which emphasised the dangers of inexperienced surgeons making major clinical decisions at night without advice from more senior staff. The difficulty in deciding when to operate for suspected acute appendicitis is balancing waiting for firmer clinical signs against possibly increasing the risk of perforation of the appendix and gangrene. ${ }^{4}$ Our study shows an increased proportion of unnecessary operations among patients presenting in the early hours but no relation between the proportion of complicated appendicitis and time of presentation. The proportion of unnecessary operations decreased with increasing time between presentation and operation with no increase in the proportion of complicated appendicitis.

At night, when faced with a choice between an unnecessary operation and repeated clinical review with the possibility of a perforated appendix later, junior doctors seem to prefer an uninflamed appendix as the lesser of two evils. This may be due to eagerness to get to bed or to various other factors including lack of sleep, worry about contacting more senior surgical staff, lack of proper training or control by senior staff, and pressure not to leave matters that may interrupt the next day's elective list.

In conclusion, the decision to operate for suspected acute appendicitis should not be made hastily in the small hours of the morning. Unless the diagnosis is clear the patient should be placed under active clinical review and the advice of more senior colleagues sought.

\footnotetext{
1 Hoffman J, Rasmussen $\mathrm{O} \emptyset$. Aids in the diagnosis of acute appendicitis. B f Surg 1989;76:774-9.

$2 \mathrm{~A}$ sound approach to the diagnosis of acute appendicitis [editorial]. Lancet 1987;24:198-9.

3 Campling EA, Devlin HB, Hoile RW, Lunn JN. The repor of the national confidential enquiry into perioperative deaths, 1990. London: National confidential enquiry into perioperative deaths, 1990.
Confidential Enquiry into Perioperative Deaths, 1992.

4 Malt RA. The perforated appendix. N Engl I Med 1986;315:1546-7.

5 Jacob ET, Bar-Nathan N, Iuchtman M. Error-rate factor in the management of appendicitis. Lancet 1975 ;i: 1032.
}

(Accepted 30 October 1992) 